\title{
Integration of Jasmonic Acid and Ethylene Into Auxin Signaling in Root Development
}

\author{
Ping $\mathrm{Xu}^{1 *}$, Ping-Xia Zhao ${ }^{2}$, Xiao-Teng Cai ${ }^{3}$, Jie-Li Mao ${ }^{2}$, Zi-Qing Miao ${ }^{2}$ and \\ Cheng-Bin Xiang ${ }^{2 *}$
}

\begin{abstract}
${ }^{1}$ Basic Forestry and Proteomics Research Center, Fujian Agriculture and Forestry University, Fuzhou, China, ${ }^{2}$ School of Life Sciences and Division of Molecular and Cell Biophysics, Hefei National Science Center for Physical Sciences at the Microscale, University of Science and Technology of China, The Innovation Academy of Seed Design, Chinese Academy of Sciences, Hefei, China, ${ }^{3}$ Beijing Advanced Innovation Center for Tree Breeding by Molecular Design, Beijing University of Agriculture, Beijing, China
\end{abstract}

\section{OPEN ACCESS}

Edited by:

Javier Brumos,

North Carolina State University,

United States

Reviewed by:

José López-Bucio,

Universidad Michoacana de San

Nicolás de Hidalgo, Mexico

Helene S. Robert,

Brno University of Technology,

Czechia

Giuseppina Falasca,

Sapienza University of Rome, Italy

${ }^{*}$ Correspondence:

Ping Xu

pingxu@mail.ustc.edu.cn

Cheng-Bin Xiang

xiangcb@ustc.edu.cn

Specialty section:

This article was submitted to Plant Development and EvoDevo,

a section of the journal

Frontiers in Plant Science

Received: 13 December 2019

Accepted: 20 February 2020

Published: 10 March 2020

Citation:

Xu P, Zhao P-X, Cai X-T, Mao J-L,

Miao Z-Q and Xiang C-B (2020)

Integration of Jasmonic Acid and Ethylene Into Auxin Signaling

in Root Development.

Front. Plant Sci. 11:271.

doi: 10.3389/fp/s.2020.00271
As sessile organisms, plants must be highly adaptable to the changing environment by modifying their growth and development. Plants rely on their underground part, the root system, to absorb water and nutrients and to anchor to the ground. The root is a highly dynamic organ of indeterminate growth with new tissues produced by root stem cells. Plants have evolved unique molecular mechanisms to fine-tune root developmental processes, during which phytohormones play vital roles. These hormones often relay environmental signals to auxin signaling that ultimately directs root development programs. Therefore, the crosstalk among hormones is critical in the root development. In this review, we will focus on the recent progresses that jasmonic acid (JA) and ethylene signaling are integrated into auxin in regulating root development of Arabidopsis thaliana and discuss the key roles of transcription factors (TFs) ethylene response factors (ERFs) and homeobox proteins in the crosstalk.

Keywords: Arabidopsis thaliana, root, auxin, jasmonic acid, ethylene, ethylene response factor, homeobox protein

\section{INTRODUCTION}

Plant root systems represent the underground organs that provide mechanical support and uptake of nutrients and water. Depending on the species and environment, root systems show a high level of morphological diversity. Improved root architecture can increase the utilization of water and nutrients, which in turn helps increase crop yield. Most dicotyledons have tap root systems, while monocotyledons have fibrous root systems. The tap root system is composed of a developed primary root, lateral roots and adventitious roots, while the fibrous root system is mainly composed of adventitious roots (Martinez-de la Cruz et al., 2015; Zhang X. et al., 2019). The development of primary roots begins from embryonic development, whereas the lateral roots are initiated from asymmetrical divisions of the pericycle founder cell of primary roots. The root system morphology or architecture (RSA) is a highly plastic trait that is influenced by numerous biotic and abiotic factors (Osmont et al., 2007). An increasing number of studies in the model plant Arabidopsis thaliana have helped to address the underlying molecular mechanisms of this plasticity (Motte et al., 2019).

Root development occurs with the concerted action of multiple plant hormones (Petricka et al., 2012). Auxin has emerged as a core player on which other plant hormones integrate to regulate root development. Auxin synthesis, transport, and signaling pathways are important for plant root development. Indole-3-acetic acid (IAA) is the main naturally occurring auxin and the biosynthetic 
pathway of IAA has been clearly understood (Zhao, 2018). L-tryptophan is the major precursor of IAA synthesis, and the rate-limiting step of tryptophan synthesis is catalyzed by anthranilate synthase (a heterocomplex consisting of ASA1/2 and ASB1) (Sun et al., 2009; Casanova-Saez and Voss, 2019). ASA1 and ASB1 are also named WEI2 (Weak Ethylene Insensitive 2) and WEI7, respectively, since they were characterized from ethylene insensitive mutants of root growth (Stepanova et al., 2005). The two-step indole-3-pyruvate (IPA) pathway is the only IAA biosynthetic pathway that has been fully elucidated, and it is also the main pathway for IAA synthesis (Zhao, 2012). TAAs (Tryptophan Aminotransferase of Arabidopsis) and YUCCAs (YUCs) are enzymes that catalyze these two steps (Mashiguchi et al., 2011; Zhao, 2012). TAA1/WEI8, like ASA1 and ASB1, was also identified from the ethylene insensitive mutant wei8 (Stepanova et al., 2008; Tao et al., 2008). Polar distribution is characteristic of auxin, which is mediated by PIN-FORMED (PIN) and AUXIN1/LIKE-AUX1 (AUX1/LAX) family members under strict regulations (Band et al., 2014; Adamowski and Friml, 2015). Localized auxin biosynthesis has been shown to play critical roles in root development as well (Zhao, 2010, 2018). Besides, the auxin signaling pathway is intensively studies recently with a focus on the fine regulation mechanisms of the IAA (INDOLEACETIC ACID-INDUCED PROTEIN) -ARF (AUXIN RESPONSE FACTOR) network (Wang and Estelle, 2014). Briefly, the auxin receptor TIR1/AFB (TRANSPORT INHIBITOR RESPONSE1/AUXIN SIGNALING F-BOX) binds to auxin causing degradation of IAA proteins that interact with ARF transcription factors (TFs) (Leyser, 2018; Gallei et al., 2019). ARFs bind to the auxin response elements (AREs) in promoters of target genes to regulate gene expression (Gallei et al., 2019).

During the stage of embryogenesis, auxin distribution patterns determine the position around which the embryonic roots start growing. In a later stage, auxin distribution patterns in and around the meristem determine root meristem activity and lateral root spacing (Motte et al., 2019). The development of lateral root is closely related to auxin, including its synthesis, transport and signal transduction (Osmont et al., 2007; Motte et al., 2019).

The gaseous phytohormone ethylene is well-known for its functions in plant maturation and senescence. In addition, numerous studies have shown that ethylene is involved in various plant growth and developmental processes, including root growth (Ruzicka et al., 2007; Swarup et al., 2007; Lewis et al., 2011; Street et al., 2015; Mao et al., 2016; Miao et al., 2018). The function of jasmonic acid (JA) in plant injury and defense responses has been thoroughly studied, and its roles in growth and development has also been widely reported (Kazan and Manners, 2013; Cai et al., 2014; Ye et al., 2019). Like other hormone signaling pathways, ethylene and JA signaling are integrated into auxin in root development, largely through TFs acting as the key crosstalk nodes.

\section{Ethylene-Auxin Crosstalk in Root Development}

Ethylene is an important regulatory signal in regulating the process of root development (Ruzicka et al., 2007; Swarup et al., 2007; Street et al., 2015). Plants produce more ethylene when exposed to external stimuli (Wang et al., 2002). Ethylene binds to ETR1(ETHYLENE RESPONSE 1) receptor family on the endoplasmic reticulum (ER) membrane, leading to inactivation of the S/T protein kinase CTR1 (CONSTITUTIVE TRIPLE RESPONSE 1), which functions to repress EIN2 (ETHYLENE INSENSITIVE 2). After detaching from CTR1, EIN2 can be cleaved to release EIN2 C-terminal (EIN2C). The EINC has two levels of regulation of EBF1/2 (EIN3-BINDING F BOX PROTEIN 1/2). On the one hand, EIN2C binds to $3^{\prime}$-UTR of $E B F 1 / 2$ in the cytoplasm to inhibit its translation (Li et al., 2015; Merchante et al., 2015), and on the other hand, EIN2C is translocated into the nucleus to promote the degradation of EBF1/2 (Qiao et al., 2012; Dolgikh et al., 2019), both leading to stabilization of EIN3/EIL1 (ETHYLENE-INSENSITIVE3LIKE1) to activate ethylene response genes. Although ethylene is best known for triggering fruit ripening, it also plays a crucial role in regulating root development. In response to ethylene or its precursor ACC (1-aminocyclopropane-1-carboxylic acid) treatment, the root of Arabidopsis seedlings shows three growth responses: rapid downregulation of cell elongation, increased root width, and induction of ectopic root hairs, which collectively will provide plants with greater anchorage and more dynamic regulation of root growth (Swarup et al., 2007).

Inhibition of root growth by ethylene depends on auxin biosynthesis, transport and signaling pathway (Ruzicka et al., 2007; Swarup et al., 2007). Ethylene up-regulates expression of auxin synthesis and transport-related genes in Arabidopsis roots, resulting in a high concentration of auxin that inhibits cell elongation (Ruzicka et al., 2007; Strader et al., 2010). Ethylene modulates the auxin transport machinery by directly or indirectly regulating the expression of auxin efflux (PINs) and influx (AUX1) carriers (Ruzicka et al., 2007). A subsequent study showed that ethylene can negatively regulate cell proliferation in addition to inhibiting cell elongation and SHY2 (SHORT HYPOCOTYL 2)/IAA3 mediated this effect in the root meristem (Street et al., 2015). It has been found that in Arabidopsis seedlings CTR1 transduces the ethylene signal to EIN2 in the root and then affects PIN2 expression to modulate the root stem cell niche maintenance (Mendez-Bravo et al., 2019). The screening experiment on the ethylene overexpression mutant eto 1 identified a small molecule named L-kynurenine (Kyn), which could inhibit ethylene-directed auxin biosynthesis and root growth by inhibiting TAAl's activity (He et al., 2011). POLARIS (PLS), encoding a predicted functional 36-amino acid peptide, is required in ethylene-mediated root inhibition through regulating auxin transport and affecting microtubule cytoskeleton dynamics (Chilley et al., 2006). The PLS expression is activated by auxin and suppressed by ethylene, and PLS peptide in turn negatively regulates the ethylene signaling pathway (Chilley et al., 2006). It was reported that ethylene can induce an oxidase named MINE, which produces pyridoxal-5' - phosphate (PNP), and PNP acts as a cofactor in TAA1/TAR-dependent auxin biosynthesis, which in turn influences ethylene-auxin crosstalk in Arabidopsis root (Kim et al., 2018).

Ethylene is involved in regulating the growth and development of not only primary roots but also lateral roots. Increased endogenous ethylene or ACC treatment activates $P I N 3 / 7$ expression thereby enhancing auxin transport 
and reducing lateral root formation (Lewis et al., 2011). Auxin signaling affects the cell division pattern of lateral root primordium by regulating the expression of the ERF (ethylene response factor) family transcription factor $P U C H I$, which is required for the proper pattern of early lateral root primordia (Hirota et al., 2007). PLS, the small peptide mentioned above, is also required in lateral roots initiation via ethylene-mediated auxin transport to the pericycle (Chilley et al., 2006).

Adventitious root initiation and development are also regulated by ethylene-auxin crosstalk. Ethylene was reported to inhibit adventitious rooting in Arabidopsis dark-grown seedlings by negatively regulating auxin biosynthesis (Veloccia et al., 2016). When applied together with IBA (indole-3-butyric acid), ethylene promotes the conversion of IBA to IAA and thus the development of adventitious roots (Veloccia et al., 2016). Ethylene-auxin crosstalk also regulates the initiation of adventitious roots near cut sites where the levels of auxin and ethylene both increase (Guan et al., 2019).

\section{JA-Auxin Crosstalk in Root Development}

Jasmonates are well-known lipid-derived compounds as key regulators in plant growth and development as well as in plant stress responses. JA participates in the regulation of root growth, seedling development, flower development, root regeneration, seed development, seed germination, tuber formation and senescence (Wasternack and Hause, 2013; Ye et al., 2019; Zhang G. et al., 2019). JA regulates root growth in many aspects, including inhibition of primary root (Chen et al., 2011), promoting lateral roots formation (Cai et al., 2014), negatively regulating adventitious roots (Gutierrez et al., 2012; Lakehal et al., 2019), and inducing root regeneration (Ye et al., 2019; Zhang G. et al., 2019). Most of these processes are achieved via cross-talking with auxin.

Root growth inhibition is one of the first discovered features of JA. By screening mutants insensitive to JAmediated root inhibition, a number of regulatory factors in the JA signaling pathway were revealed, such as JAR1 (JASMONATE RESISTANT 1) (Staswick et al., 1992), MYC2/JAI1 (JASMONATE INSENSITIVE 1) (Berger et al., 1996), and COI1 (CORONATINE INSENSITIVE 1) (Feys et al., 1994). JA inhibits root elongation by reducing both cell counts and cell dimension, suggesting that JA-induced primary root growth inhibition is a complicated process involving diverse cellular processes in different root tissues (Chen et al., 2011, 2012). JA-mediated inhibition of root development is auxindependent (Wasternack and Hause, 2013). JA activates MYC2, leading to the repression of PLT1 (PLETHORA1) and PLT2 in root stem cell niche (Chen et al., 2011). PLTs encodes members of the AP2/EREBP transcription factor family and are key effectors for the establishment of the stem cell niche during embryonic pattern formation. They respond to auxin accumulation and this response depends on auxin-responsive TFs. Therefore, PLTs serve as a key node for JA-auxin crosstalk in regulating the maintenance of the stem cell niche in roots (Chen et al., 2011).

Jasmonic acid is also involved in regulating lateral roots development. In response to methyl jasmonate (MeJA) treatment, Arabidopsis wild type produces more lateral roots, while the mutant asa1-1 does not produce lateral roots (Sun et al., 2009). The JA receptor COI1 plays a critical role in the formation and even distribution of lateral roots (RayaGonzalez et al., 2012). In the coil-1 mutant, the lateral roots displayed uneven distribution and JA failed to induce more lateral roots (Raya-Gonzalez et al., 2012). In the root, MeJA activates the transcription of $A S A 1$ and several other auxin biosynthesis-related genes, such as YUCCA2 (Cheng et al., 2006), ASB1 (Stepanova et al., 2005), and NITRILASE 3 (NIT3) (Kutz et al., 2002). JA failed to increase lateral root initiation in mutants with disrupted auxin signaling, like slr1 (iaa14) and arf7/19 double mutant (Sun et al., 2009), which further supports that JA-induced lateral root formation is auxin-dependent. Activated expression of the transcription regulator HDG11 (HOMEODOMAIN GLABROUS11) increases the level of JA in the roots by directly up-regulating the expression of several genes encoding JA biosynthetic enzymes, resulting in enhanced auxin signaling and lateral root formation (Cai et al., 2015). MeJA can also induce YUC8 and YUC9 expression and thus participate in auxin-mediated primary root growth and lateral root initiation (Hentrich et al., 2013).

Jasmonic acid can exert negative effects on adventitious root formation (Gutierrez et al., 2012). ARF6, ARF8, and ARF17 act upstream of Gretchen Hagen3.3 (GH3.3), GH3.5, and GH3.6. These three GH3s inactive JA by conjugating JA to amino acids Asp, Met, and Trp, and therefore promote adventitious rooting (Gutierrez et al., 2012). The effect of JA in adventitious root development depends on experimental conditions. At low submicromolar concentrations, MeJA has been shown to promote adventitious root development when applied together with IBA, and this process does not involve regulation of ARF6 or ARF8 expression (Fattorini et al., 2018).

It is well known that plants can regenerate tissues and even complete organs after damage. Recently, Zhou et al. (2019) reported that the synergy between jasmonate and auxin signaling pathways promotes root regeneration by activating root stem cells (Zhou et al., 2019). In this process, JA induces ERF109, CYCLIN D6;1 (CYCD6;1), and ERF115 expression to activate stem cell and promote tissue regeneration. Auxin also activates key regeneration regulators of this pathway (Zhou et al., 2019).

\section{TFs Involved in JA-Auxin and Ethylene-Auxin Crosstalk}

Transcription factors are specific implementers of numerous regulation processes. Each hormone crosstalk involves many important TFs. Here we focus on ERF family and HD-ZIP TFs in JA-auxin and ethylene-auxin crosstalk.

Ethylene response factor family TFs are plant specific and involve in a variety of plant development processes and stress responses. Many ERFs are responsive to ethylene. ERF1 negatively regulates primary root elongation in an auxin biosynthesis-dependent manner. Being downstream and a direct target of EIN3, ERF1 activates ASA1 by binding to GCCGCC motifs (GCC-boxes) in the promoter of ASA1. The up-regulation of ASA1 increases auxin biosynthesis, promotes auxin accumulation in root tip, and consequently suppresses root 
elongation (Mao et al., 2016). Therefore, ERF1 acts as a critical crosstalk joint connecting ethylene and auxin in regulating primary root elongation (Figure 1).

HOMEOBOX PROTEIN52 (HB52) belongs to the HD-ZIP transcription factor family. The HD-ZIP transcription factor family only found in plants with 47 members in Arabidopsis. According to protein structures and functions, HD-ZIP family members can be divided into four subfamilies (I-IV), and HB52 belongs to the HD-ZIP I subfamily (Ariel et al., 2007). HD-ZIP I family genes are responsive to external stimuli like drought, high temperature, osmotic stress, and lights. HB52 was identified from the study of ethylene-mediated root inhibition (Mao et al., 2016). HB52 is highly expressed in roots and is responsive to the ACC treatment as a direct target of EIN3. HB52 regulates primary root elongation through affecting auxin transport. HB52 binds to the homeodomainbinding cis-elements in the promoters of PIN2 and WAG1/2 to activate their expression (Figure 1). WAG1/2, closely related to PINOID, can phosphorylate PIN2 to increase its auxin efflux carrier ability (Willige et al., 2012). Therefore, HB52 serves as another important crosstalk node between ethylene and auxin to regulate root elongation (Miao et al., 2018). Together, ERF1 and HB52 constitute the ethylene-responsive modules for auxin biosynthesis and transport, respectively, in root elongation regulation.

ERF109 is another member of the ERF family and responsive to the JA signaling pathway. ERF109 binds to GCC-boxes in the promoter regions of its target genes. Under normal conditions,

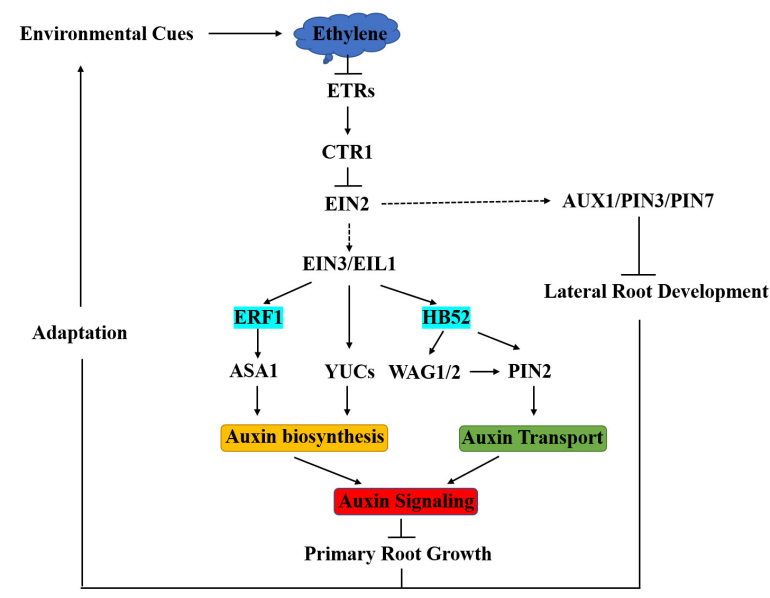

FIGURE 1 | Integration of Ethylene into Auxin Signaling in Arabidopsis Root Development. Environmental cues trigger the biosynthesis of ethylene in Arabidopsis, and then ethylene binds to ETR receptors to inactivate CTR1, which functions to repress EIN2. When EIN2 is released by CTR1, it can be cleaved and then helps to stabilize EIN3/EIL1, leading to the activation of downstream transcriptional cascades. Ethylene inhibits primary root growth by regulating auxin biosynthesis, transport, and signaling. ERF1 and HB52 function as crosstalk nodes between ethylene and auxin in this process. An increase in endogenous ethylene enhances auxin transport and reduces lateral root formation depending on AUX1, PIN3, and PIN7. The ERF1 and $\mathrm{HB} 52$ regulatory modules are part of the molecular mechanisms in the adaptive response of root growth to environmental cues.
ERF109 is expressed at a very low level in roots. After MeJA treatment, the transcription level of ERF109 was significantly induced in both roots and shoots, especially in the lateral root primordium region and the tip and base of lateral roots (Cai et al., 2014). Genetic analyses showed that ERF109 positively regulates lateral root formation through upregulating auxin biosynthesis. In vitro and in vivo experiments showed that ERF109 binds to the GCC-boxes in ASA1 and YUC2 promoters and directly activates their expression, leading to increased auxin biosynthesis and accumulation in the root (Cai et al., 2014). Thus, ERF109 serves as an important crosstalk node between JA and auxin signaling (Figure 2). Recently, three research groups independently reported that ERF109 has a novel function in plant regeneration depending on its roles in upregulating ASA1 expression (Ye et al., 2019; Zhang G. et al., 2019) or activating ERF115 and CYCD6;1 (Zhou et al., 2019).

ERF1, ERF109, and HB52 are representative TFs involved in the crosstalk of JA-auxin and ethylene-auxin signaling pathways in regulating root development. Other TFs participated in the processes are yet to be identified.

\section{CONCLUSION AND PERSPECTIVES}

Crosstalk between hormone signaling are fundamental process in plant development, yet the underlying mechanisms are far from clear. In this review, we summarized recent advances on the understanding of ethylene and JA integration into auxin signaling in the regulation of root development.

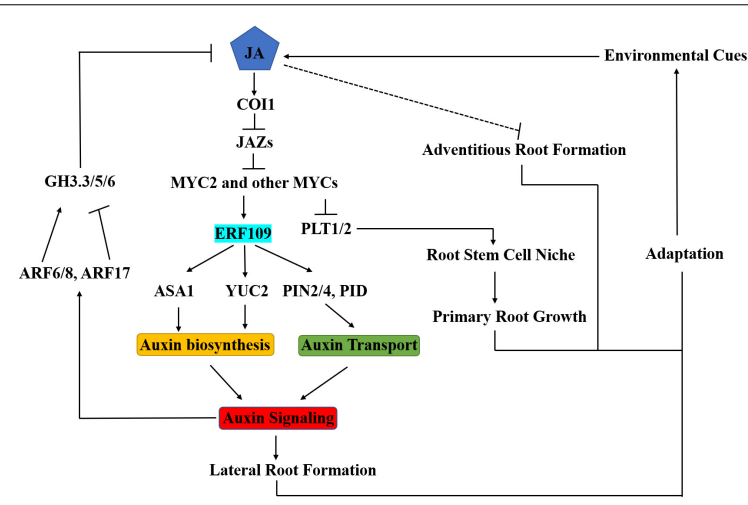

FIGURE 2 | Integration of JA into Auxin Signaling in Arabidopsis Root Development. Plants generate JA in response to environmental cues. COI1 receptor perceives JA, and then recruits JAZs subjected to degradation. Subsequently, MYC2 can activate transcription of early JA-responsive genes. JA promotes lateral root formation by regulating auxin biosynthesis (via ASA1 and YUC2) and transport (via PID and PIN2/4). Transcription factor ERF109 functions as a key crosstalk node in this process. JA inhibits primary root development by repressing the expression of PLT1 and PLT2. Auxin modulates JA homeostasis by regulating GH3.3/5/6 through ARF6/8/17, then influences adventitious root formation. Therefore, the ERF109 regulatory module plays critical roles in the growth and development of lateral, primary and adventitious roots in the adaptive response of the root system to environmental factors. 
Auxin plays the central role in regulating root development. Plant roots constantly perceive environmental cues and generate hormonal signals in order to adjust developmental programs for better adaptation to the changing surroundings. JA and ethylene are two representative hormones in plants responding to environmental changes. These two hormonal signals can be relayed to auxin signaling, the master regulator of root development. In the signal relay, TFs play critical roles to integrate other hormonal signal into auxin signaling through modulating auxin biosynthesis (for example, ERF1 and ERF109) or auxin transport (for example, HB52) to fine-tune the regulation of primary root growth and/or lateral root formation.

To unravel the complete network of JA-auxin and ethyleneauxin crosstalks in root development, we need to identify the more components involved in these processes, as well as understand the spatial-temporal relationships between these components. Some attempts have been recently made by identifying the root epidermis cells where the interaction between

\section{REFERENCES}

Adamowski, M., and Friml, J. (2015). PIN-dependent auxin transport: action, regulation, and evolution. Plant Cell 27, 20-32. doi: 10.1105/tpc.114.134874

Ariel, F. D., Manavella, P. A., Dezar, C. A., and Chan, R. L. (2007). The true story of the HD-Zip family. Trends Plant Sci. 12, 419-426. doi: 10.1016/j.tplants.2007. 08.003

Band, L. R., Wells, D. M., Fozard, J. A., Ghetiu, T., French, A. P., Pound, M. P., et al. (2014). Systems analysis of auxin transport in the Arabidopsis root apex. Plant Cell 26, 862-875. doi: 10.1105/tpc.113.119495

Berger, S., Bell, E., and Mullet, J. E. (1996). Two Methyl jasmonate-insensitive mutants show altered expression of atvsp in response to methyl jasmonate and wounding. Plant Physiol. 111, 525-531. doi: 10.1104/pp.111.2.525

Brumos, J., Robles, L. M., Yun, J., Vu, T. C., Jackson, S., Alonso, J. M., et al. (2018). Local auxin biosynthesis is a key regulator of plant development. Dev. Cell 47:e305.

Cai, X. T., Xu, P., Wang, Y., and Xiang, C. B. (2015). Activated expression of AtEDT1/HDG11 promotes lateral root formation in Arabidopsis mutant edt1 by upregulating jasmonate biosynthesis. J. Integr. Plant Biol. 57, 1017-1030. doi: $10.1111 /$ jipb.12347

Cai, X. T., Xu, P., Zhao, P. X., Liu, R., Yu, L. H., and Xiang, C. B. (2014). Arabidopsis ERF109 mediates cross-talk between jasmonic acid and auxin biosynthesis during lateral root formation. Nat. Commun. 5:5833. doi: 10.1038/ ncomms6833

Casanova-Saez, R., and Voss, U. (2019). Auxin metabolism controls developmental decisions in land plants. Trends Plant Sci. 24, 741-754. doi: 10.1016/j.tplants. 2019.05.006

Chen, Q., Sun, J., Zhai, Q., Zhou, W., Qi, L., Xu, L., et al. (2011). The basic helix-loop-helix transcription factor MYC2 directly represses PLETHORA expression during jasmonate-mediated modulation of the root stem cell niche in Arabidopsis. Plant Cell 23, 3335-3352. doi: 10.1105/tpc.111.08 9870

Chen, R., Jiang, H., Li, L., Zhai, Q., Qi, L., Zhou, W., et al. (2012). The Arabidopsis mediator subunit MED25 differentially regulates jasmonate and abscisic acid signaling through interacting with the MYC2 and ABI5 transcription factors. Plant Cell 24, 2898-2916. doi: 10.1105/tpc.112.098277

Cheng, Y., Dai, X., and Zhao, Y. (2006). Auxin biosynthesis by the YUCCA flavin monooxygenases controls the formation of floral organs and vascular tissues in Arabidopsis. Genes Dev. 20, 1790-1799. doi: 10.1101/gad.141 5106

Chilley, P. M., Casson, S. A., Tarkowski, P., Hawkins, N., Wang, K. L., Hussey, P. J., et al. (2006). The POLARIS peptide of Arabidopsis regulates auxin transport and root growth via effects on ethylene signaling. Plant Cell 18, 3058-3072. doi: $10.1105 /$ tpc. 106.040790 ethylene and auxin takes place (Vaseva et al., 2018; MendezBravo et al., 2019). Moreover, local auxin biosynthesis is critical in ethylene-auxin crosstalk (Brumos et al., 2018). With the advance of new technology such as single-cell sequencing and high-resolution microscope, in-depth details in the crosstalk will be revealed.

\section{AUTHOR CONTRIBUTIONS}

PX and C-BX conceived the mini-review. PX wrote the minireview. All authors contributed to the discussion and revision.

\section{FUNDING}

This work was funded by the National Natural Science Foundation of China (Grant 31600195 to PX).

Dolgikh, V. A., Pukhovaya, E. M., and Zemlyanskaya, E. V. (2019). shaping ethylene response: the role of EIN3/EIL1 transcription factors. Front. Plant Sci. 10:1030. doi: 10.3389/fpls.2019.01030

Fattorini, L., Hause, B., Gutierrez, L., Veloccia, A., Della Rovere, F., Piacentini, D., et al. (2018). Jasmonate promotes auxin-induced adventitious rooting in dark-grown Arabidopsis thaliana seedlings and stem thin cell layers by a crosstalk with ethylene signalling and a modulation of xylogenesis. BMC Plant Biol. 18:182. doi: 10.1186/s12870-018-1392-4

Feys, B., Benedetti, C. E., Penfold, C. N., and Turner, J. G. (1994). Arabidopsis mutants selected for resistance to the phytotoxin coronatine are male sterile, insensitive to Methyl jasmonate, and resistant to a bacterial pathogen. Plant Cell 6, 751-759. doi: 10.1105/tpc.6.5.751

Gallei, M., Luschnig, C., and Friml, J. (2019). Auxin signalling in growth: schrodinger's cat out of the bag. Curr. Opin. Plant Biol. 53, 43-49. doi: 10.1016/ j.pbi.2019.10.003

Guan, L., Tayengwa, R., Cheng, Z. M., Peer, W. A., Murphy, A. S., and Zhao, M. (2019). Auxin regulates adventitious root formation in tomato cuttings. BMC Plant Biol. 19:435. doi: 10.1186/s12870-019-2002-9

Gutierrez, L., Mongelard, G., Flokova, K., Pacurar, D. I., Novak, O., Staswick, P., et al. (2012). Auxin controls Arabidopsis adventitious root initiation by regulating jasmonic acid homeostasis. Plant Cell 24, 2515-2527. doi: 10.1105/ tpc.112.099119

He, W., Brumos, J., Li, H., Ji, Y., Ke, M., Gong, X., et al. (2011). A small-molecule screen identifies L-kynurenine as a competitive inhibitor of TAA1/TAR activity in ethylene-directed auxin biosynthesis and root growth in Arabidopsis. Plant Cell 23, 3944-3960. doi: 10.1105/tpc.111.089029

Hentrich, M., Bottcher, C., Duchting, P., Cheng, Y., Zhao, Y., Berkowitz, O., et al. (2013). The jasmonic acid signaling pathway is linked to auxin homeostasis through the modulation of YUCCA8 and YUCCA9 gene expression. Plant J. 74, 626-637. doi: 10.1111/tpj.12152

Hirota, A., Kato, T., Fukaki, H., Aida, M., and Tasaka, M. (2007). The auxinregulated AP2/EREBP gene PUCHI is required for morphogenesis in the early lateral root primordium of Arabidopsis. Plant Cell 19, 2156-2168. doi: 10.1105/ tpc.107.050674

Kazan, K., and Manners, J. M. (2013). MYC2: the master in action. Mol. Plant 6, 686-703. doi: $10.1093 / \mathrm{mp} / \mathrm{sss} 128$

Kim, G., Jang, S., Yoon, E. K., Lee, S. A., Dhar, S., Kim, J., et al. (2018). Involvement of Pyridoxine/Pyridoxamine 5'-Phosphate Oxidase (PDX3) in ethylene-induced auxin biosynthesis in the Arabidopsis root. Mol. Cells 41, 1033-1044. doi: 10.14348/molcells.2018.0363

Kutz, A., Muller, A., Hennig, P., Kaiser, W. M., Piotrowski, M., and Weiler, E. W. (2002). A role for nitrilase 3 in the regulation of root morphology in sulphurstarving Arabidopsis thaliana. Plant J. 30, 95-106. doi: 10.1046/j.1365-313x. 2002.01271.x 
Lakehal, A., Chaabouni, S., Cavel, E., Le Hir, R., Ranjan, A., Raneshan, Z., et al. (2019). A molecular framework for the control of adventitious rooting by the TIR1/AFB2-Aux/IAA-dependent auxin signaling in Arabidopsis. Mol. Plant 12, 1499-1514. doi: 10.1016/j.molp.2019.09.001

Lewis, D. R., Negi, S., Sukumar, P., and Muday, G. K. (2011). Ethylene inhibits lateral root development, increases IAA transport and expression of PIN3 and PIN7 auxin efflux carriers. Development 138, 3485-3495. doi: 10.1242/dev. 065102

Leyser, O. (2018). Auxin signaling. Plant Physiol. 176, 465-479.

Li, W., Ma, M., Feng, Y., Li, H., Wang, Y., Ma, Y., et al. (2015). EIN2-directed translational regulation of ethylene signaling in Arabidopsis. Cell 163, 670-683. doi: 10.1016/j.cell.2015.09.037

Mao, J. L., Miao, Z. Q., Wang, Z., Yu, L. H., Cai, X. T., and Xiang, C. B. (2016). Arabidopsis ERF1 mediates cross-talk between ethylene and auxin biosynthesis during primary root elongation by regulating ASA1 expression. PLoS Genet. 12:e1005760. doi: 10.1371/journal.pgen.1005760

Martinez-de la Cruz, E., Garcia-Ramirez, E., Vazquez-Ramos, J. M., Reyes de la Cruz , H., and Lopez-Bucio, J. (2015). Auxins differentially regulate root system architecture and cell cycle protein levels in maize seedlings. J. Plant Physiol. 176, 147-156. doi: 10.1016/j.jplph.2014.11.012

Mashiguchi, K., Tanaka, K., Sakai, T., Sugawara, S., Kawaide, H., Natsume, M., et al. (2011). The main auxin biosynthesis pathway in Arabidopsis. Proc. Natl. Acad. Sci. U.S.A. 108, 18512-18517. doi: 10.1073/pnas. 1108434108

Mendez-Bravo, A., Ruiz-Herrera, L. F., Cruz-Ramirez, A., Guzman, P., MartinezTrujillo, M., Ortiz-Castro, R., et al. (2019). CONSTITUTIVE TRIPLE RESPONSE1 and PIN2 act in a coordinate manner to support the indeterminate root growth and meristem cell proliferating activity in Arabidopsis seedlings. Plant Sci. 280, 175-186. doi: 10.1016/j.plantsci.2018.11.019

Merchante, C., Brumos, J., Yun, J., Hu, Q., Spencer, K. R., Enriquez, P., et al. (2015). Gene-specific translation regulation mediated by the hormonesignaling molecule EIN2. Cell 163, 684-697. doi: 10.1016/j.cell.2015.09.036

Miao, Z. Q., Zhao, P. X., Mao, J. L., Yu, L. H., Yuan, Y., Tang, H., et al. (2018), HOMEOBOX PROTEIN52 mediates the crosstalk between ethylene and auxin signaling during primary root elongation by modulating auxin transportrelated gene expression. Plant Cell 30, 2761-2778. doi: 10.1105/tpc.18. 00584

Motte, H., Vanneste, S., and Beeckman, T. (2019). Molecular and environmental regulation of root development. Annu. Rev. Plant Biol. 70, 465-488. doi: 10. 1146/annurev-arplant-050718-100423

Osmont, K. S., Sibout, R., and Hardtke, C. S. (2007). Hidden branches: developments in root system architecture. Annu. Rev. Plant Biol. 58, 93-113. doi: 10.1146/annurev.arplant.58.032806.104006

Petricka, J. J., Winter, C. M., and Benfey, P. N. (2012). Control of Arabidopsis root development. Annu. Rev. Plant Biol. 63, 563-590. doi: 10.1146/annurevarplant-042811-105501

Qiao, H., Shen, Z., Huang, S. S., Schmitz, R. J., Urich, M. A., Briggs, S. P., et al. (2012). Processing and subcellular trafficking of ER-tethered EIN2 control response to ethylene gas. Science 338, 390-393. doi: 10.1126/science.1225974

Raya-Gonzalez, J., Pelagio-Flores, R., and Lopez-Bucio, J. (2012). The jasmonate receptor COI1 plays a role in jasmonate-induced lateral root formation and lateral root positioning in Arabidopsis thaliana. J. Plant Physiol. 169, 1348-1358. doi: 10.1016/j.jplph.2012.05.002

Ruzicka, K., Ljung, K., Vanneste, S., Podhorska, R., Beeckman, T., Friml, J., et al. (2007). Ethylene regulates root growth through effects on auxin biosynthesis and transport-dependent auxin distribution. Plant Cell 19, 2197-2212. doi: $10.1105 /$ tpc. 107.052126

Staswick, P. E., Su, W., and Howell, S. H. (1992). Methyl jasmonate inhibition of root growth and induction of a leaf protein are decreased in an Arabidopsis thaliana mutant. Proc. Natl. Acad. Sci. U.S.A. 89, 6837-6840. doi: 10.1073/pnas. 89.15.6837

Stepanova, A. N., Hoyt, J. M., Hamilton, A. A., and Alonso, J. M. (2005). A Link between ethylene and auxin uncovered by the characterization of two rootspecific ethylene-insensitive mutants in Arabidopsis. Plant Cell 17, 2230-2242. doi: 10.1105/tpc. 105.033365

Stepanova, A. N., Robertson-Hoyt, J., Yun, J., Benavente, L. M., Xie, D. Y., Dolezal, K., et al. (2008). TAA1-mediated auxin biosynthesis is essential for hormone crosstalk and plant development. Cell 133, 177-191. doi: 10.1016/j.cell.2008.01. 047
Strader, L. C., Chen, G. L., and Bartel, B. (2010). Ethylene directs auxin to control root cell expansion. Plant J. 64, 874-884. doi: 10.1111/j.1365-313X.2010. 04373.x

Street, I. H., Aman, S., Zubo, Y., Ramzan, A., Wang, X., Shakeel, S. N., et al. (2015). Ethylene Inhibits Cell Proliferation of the Arabidopsis Root Meristem. Plant Physiol. 169, 338-350. doi: 10.1104/pp.15.00415

Sun, J., Xu, Y., Ye, S., Jiang, H., Chen, Q., Liu, F., et al. (2009). Arabidopsis ASA1 is important for jasmonate-mediated regulation of auxin biosynthesis and transport during lateral root formation. Plant Cell 21, 1495-1511. doi: $10.1105 /$ tpc. 108.064303

Swarup, R., Perry, P., Hagenbeek, D., Van Der Straeten, D., Beemster, G. T., Sandberg, G., et al. (2007). Ethylene upregulates auxin biosynthesis in Arabidopsis seedlings to enhance inhibition of root cell elongation. Plant Cell 19, 2186-2196. doi: 10.1105/tpc.107.052100

Tao, Y., Ferrer, J. L., Ljung, K., Pojer, F., Hong, F., Long, J. A., et al. (2008). Rapid synthesis of auxin via a new tryptophan-dependent pathway is required for shade avoidance in plants. Cell 133, 164-176. doi: 10.1016/j.cell.2008.01.049

Vaseva, I. I., Qudeimat, E., Potuschak, T., Du, Y., Genschik, P., Vandenbussche, F., et al. (2018). The plant hormone ethylene restricts Arabidopsis growth via the epidermis. Proc. Natl. Acad. Sci. U.S.A. 115, E4130-E4139. doi: 10.1073/pnas. 1717649115

Veloccia, A., Fattorini, L., Della Rovere, F., Sofo, A., D’Angeli, S., Betti, C., et al. (2016). Ethylene and auxin interaction in the control of adventitious rooting in Arabidopsis thaliana. J. Exp. Bot. 67, 6445-6458. doi: 10.1093/jxb/erw415

Wang, K. L. C., Li, H., and Ecker, J. R. (2002). ethylene biosynthesis and signaling networks. Plant Cell 14, S131-S151.

Wang, R., and Estelle, M. (2014). Diversity and specificity: auxin perception and signaling through the TIR1/AFB pathway. Curr. Opin. Plant Biol. 21, 51-58. doi: 10.1016/j.pbi.2014.06.006

Wasternack, C., and Hause, B. (2013). Jasmonates: biosynthesis, perception, signal transduction and action in plant stress response, growth and development. An update to the 2007 review in Annals of Botany. Ann. Bot. 111, 1021-1058. doi: $10.1093 / \mathrm{aob} / \mathrm{mct} 067$

Willige, B. C., Ogiso-Tanaka, E., Zourelidou, M., and Schwechheimer, C. (2012). WAG2 represses apical hook opening downstream from gibberellin and PHYTOCHROME INTERACTING FACTOR 5. Development 139, 4020-4028. doi: $10.1242 / \mathrm{dev} .081240$

Ye, B. B., Shang, G. D., Pan, Y., Xu, Z. G., Zhou, C. M., Mao, Y. B., et al. (2019). AP2/ERF transcription factors integrate age and wound signals for root regeneration. Plant Cell 32, 226-241. doi: 10.1105/tpc.19.00378

Zhang, G., Zhao, F., Chen, L., Pan, Y., Sun, L., Bao, N., et al. (2019). Jasmonatemediated wound signalling promotes plant regeneration. Nat. Plants 5, 491497. doi: 10.1038/s41477-019-0408-x

Zhang, X., Wang, B., Zhao, Y., Zhang, J., and Li, Z. (2019). Auxin and GA signaling play important roles in the maize response to phosphate deficiency. Plant Sci. 283, 177-188. doi: 10.1016/j.plantsci.2019.02.011

Zhao, Y. (2010). Auxin biosynthesis and its role in plant development. Annu. Rev. Plant Biol. 61, 49-64. doi: 10.1146/annurev-arplant-042809-112308

Zhao, Y. (2012). Auxin biosynthesis: a simple two-step pathway converts tryptophan to indole-3-acetic acid in plants. Mol. Plant 5, 334-338. doi: 10. 1093/mp/ssr104

Zhao, Y. (2018). Essential roles of local auxin biosynthesis in plant development and in adaptation to environmental changes. Annu. Rev. Plant Biol. 69, 417435. doi: 10.1146/annurev-arplant-042817-040226

Zhou, W., Lozano-Torres, J. L., Blilou, I., Zhang, X., Zhai, Q., Smant, G., et al. (2019). A jasmonate signaling network activates root stem cells and promotes regeneration. Cell 177, 942.e14-956e.14. doi: 10.1016/j.cell.2019.03.006

Conflict of Interest: The authors declare that the research was conducted in the absence of any commercial or financial relationships that could be construed as a potential conflict of interest.

Copyright (c) $2020 \mathrm{Xu}$, Zhao, Cai, Mao, Miao and Xiang. This is an open-access article distributed under the terms of the Creative Commons Attribution License (CC BY). The use, distribution or reproduction in other forums is permitted, provided the original author(s) and the copyright owner(s) are credited and that the original publication in this journal is cited, in accordance with accepted academic practice. No use, distribution or reproduction is permitted which does not comply with these terms. 